\title{
CHASM (CHerenkov Air Shower Model): Simulating the Cherenkov Profiles of Cosmic Ray Air Showers
}

\author{
Isaac Buckland* and Douglas Bergman on behalf of the Telescope Array \\ Collaboration $^{\dagger}$ \\ ${ }^{a}$ Dept. of Physics \& Astronomy and High Energy Astrophysics Inst., \\ University of Utah, Utah, USA \\ E-mail: ikepc1@gmail.com, bergman@physics.utah.edu
}

Reconstruction of an EAS seen using non-imaging Cherenkov detectors requires simulating the Cherenkov yield of many EAS's with given shower parameters. Since Shower Universality parameterizes both the angular distribution and energy distribution of charged particles within a shower, one can calculate the Cherenkov photon yield (at a fixed point) from the Cherenkov cones of electrons. In this work, we compare both the CWLD (Cherenkov Width Lateral Distribution) and arrival time distributions from Cherenkov universality calculations with those from CORSIKA iact (imaging atmospheric Cherenkov telescope) simulations. Since universality calculations are much less computationally expensive than shower simulation programs like CORSIKA, reconstruction could be accomplished more efficiently using Cherenkov data.

$37^{\text {th }}$ International Cosmic Ray Conference (ICRC 2021)

July 12th - 23rd, 2021

Online - Berlin, Germany

\footnotetext{
${ }^{*}$ Presenter

${ }^{\dagger}$ A complete list of collaborators see Pos(ICRC2021)
} 


\section{Introduction}

The simulation of cosmic ray air showers is a computationally expensive process. This work illustrates how shower universality can be used to simulate the Cherenkov light profile of cosmic ray air showers. The CHASM python module was developed for all shower geometries and profiles, and is intended to be a computationally efficient alternative to Monte Carlo air shower simulation packages like CORSIKA. CHASM is still in development. This work is meant to showcase its current progress and functionality. We present simulations of Cherenkov distributions from various shower types, as well as comparisons to CORSIKA's IACT (Imaging Atmospheric Cherenkov Telescope) extension.

\section{What is Shower Universality?}

Shower universality is the principle that properties of secondary particles in an air shower such as propagation direction, energy, and lateral spread can be represented by universal parameterized distributions [1] [2]. These parameterizations vary as a shower develops and take into account secondary particle properties such as energy (i.e. the directional distribution of particles in a given energy interval will be narrower for higher energies). Charged particles in a shower will produce Cherenkov light in a cone determined by particle energy and the atmospheric index of refraction in which they propagate. Published fits to secondary particle distributions were used to create a table of Cherenkov angular distributions at various atmospheric indices of refraction and stages of shower development. CHASM generates a shower profile in the Earth's atmosphere, then accesses the Cherenkov table to calculate the photon yield at user defined locations.

\section{NICHE (Non Imaging CHErenkov) and TALE (Telescope Array Low Energy)}

An efficient Cherenkov signal simulation will enable a hybrid analysis of the low-energy cosmic ray spectrum using data from both the TALE fluorescence detector and the NICHE (Non Imaging CHErenkov) detectors near the Middle Drum telescope site. In Figure 1 we see the NICHE array relative to the position of the Middle Drum observatory, lying directly beneath TALE's field of view. The NICHE detectors are simple light buckets consisting of a PMT (Photo-Multiplier Tube) sensitive to Cherenkov light between 300 and $450 \mathrm{~nm}$. There is currently an analysis being performed looking for coincidence events between NICHE and TALE. In Figure 2, we see a sample NICHE event with the Cherenkov signal sweeping diagonally through the array.

\section{Universality vs. CORSIKA}

To demonstrate the veracity of the Cherenkov universality model, we generated a Cherenkov light profile using universality, and compared it to a profile generated by CORSIKA's IACT extension. This downward proton shower has a primary energy of $108 \mathrm{GeV}, X_{\max }$ at $863 \mathrm{~g} / \mathrm{cm} 2, N_{\max }$ of 67 million particles, and a polar angle of 10 degrees. $X_{\max }$ and $N_{\max }$ refer to the atmospheric depth at shower maximum and the maximum number of secondary shower particles, respectively. IACT counters were defined at the altitude of the lowest Telescope Array NICHE counter (1564 m above 

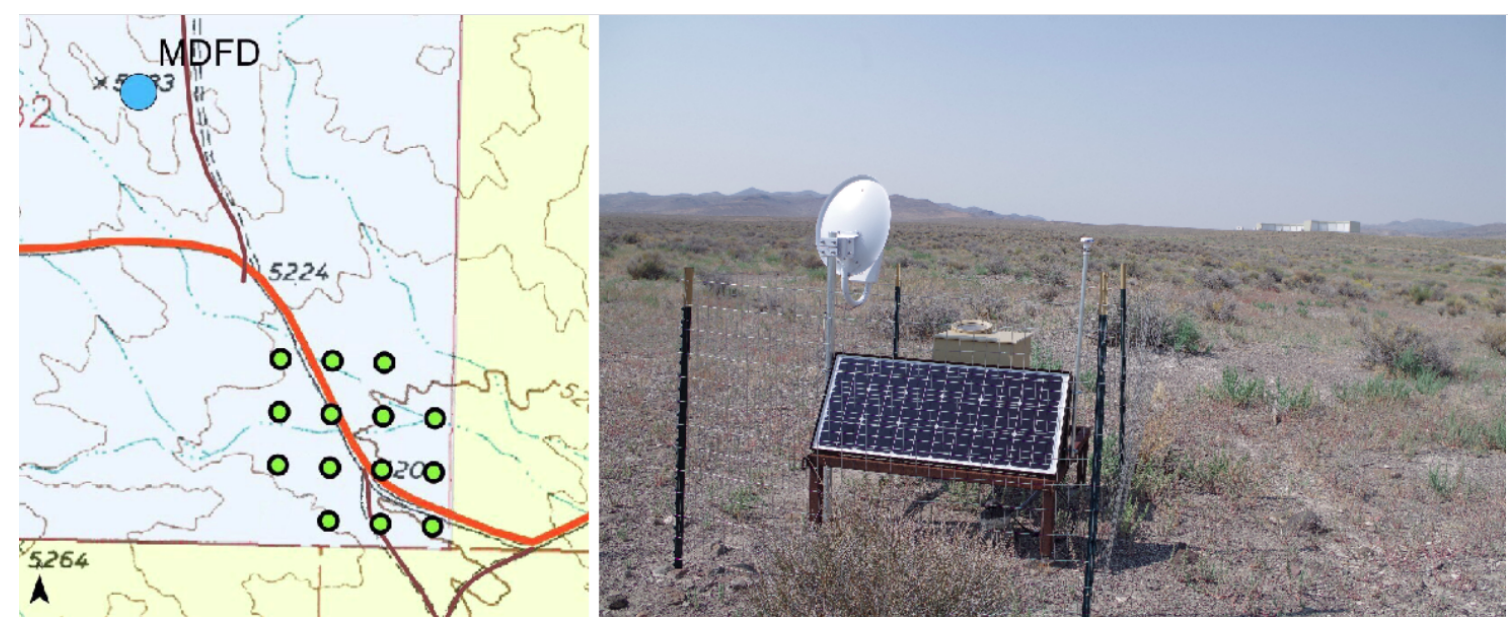

Figure 1: Left: the orientation of the NICHE array with respect to the Middle Drum observatory. Right: One of the NICHE counters with Middle Drum in the background

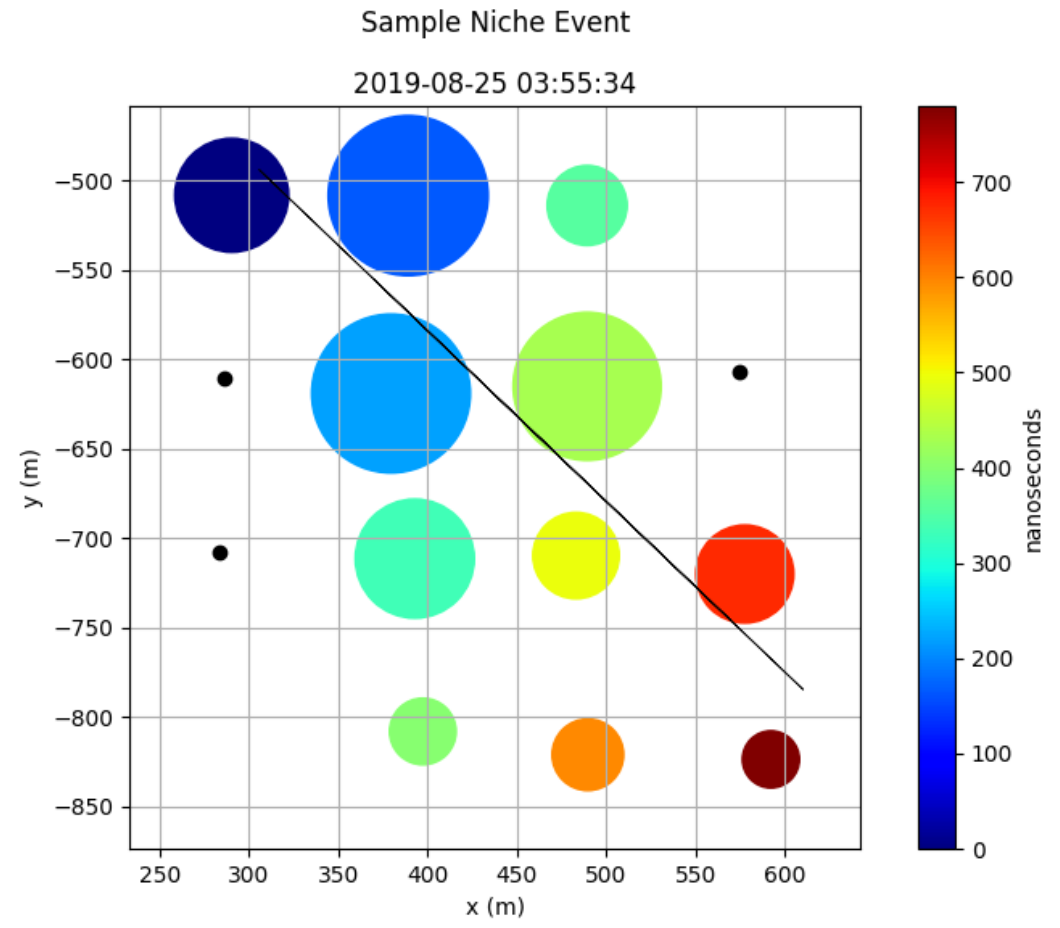

Figure 2: Event display for NICHE event. The circle size of each triggered detector is scaled by the signal pulse area. The time of each trigger is represented by its color. 

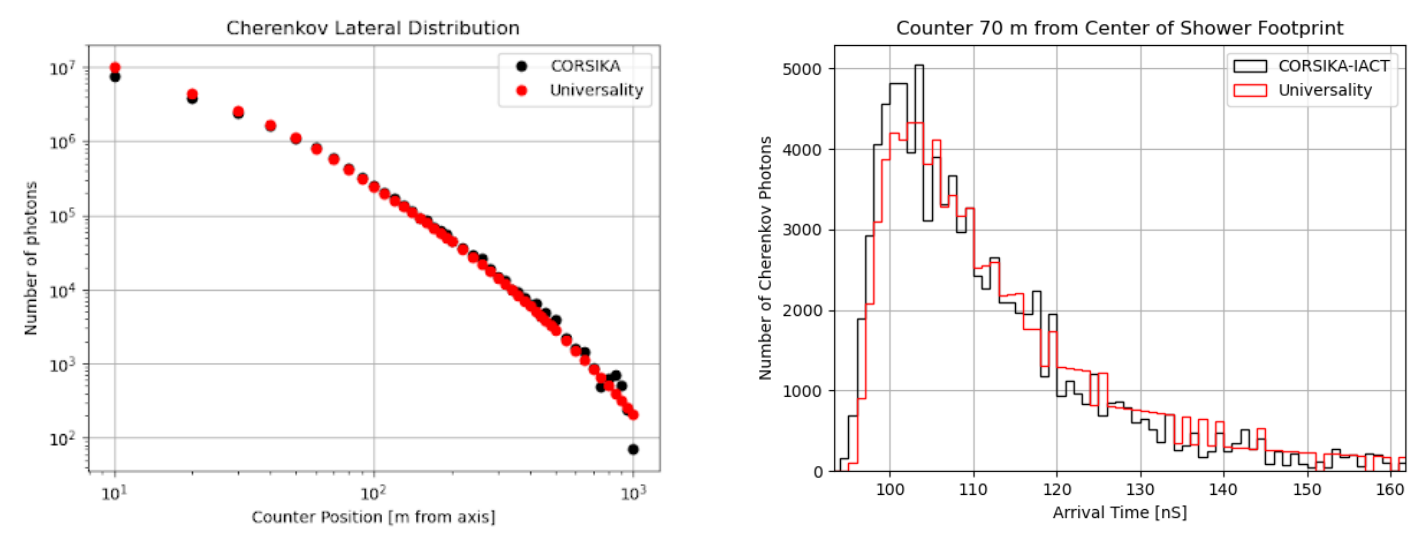

Figure 3: Left: Comparison of Cherenkov lateral distribution from both CORSIKA and universality. Right: Comparison of arrival time distribution from both CORSIKA and universality.

sea level). The total number of photons collected at increasing distances from the shower core are shown in Figure 3, and the arrival time distributions are compared for a counter $70 \mathrm{~m}$ from the shower core in Figure 3.

\section{Hypothetical Tau Primary Upward Air Shower}

The first iteration of CHASM is being tested for implementation in nuSpaceSim, a comprehensive neutrino simulation package for space-based and suborbital experiments [3]. Tau neutrinos skimming the Earth may interact via the charged-current interaction in the Earth's crust. The resulting tau particle leaves the Earth and decays, serving as the primary particle in an upward going air shower. For this demo, an upward shower profile was generated using the Gaisser-Hillas function with an $X_{\max }$ of $785 \mathrm{~g} / \mathrm{cm} 2$, an $N_{\max }$ of 80 million particles, and a first-interaction height of $10 \mathrm{Km}$. The resulting Cherenkov light was calculated at an array of locations normal to the shower axis at an altitude of $525 \mathrm{~km}$. Figure 4 shows the shower orientation, as well as the $\mathrm{x}$ and $\mathrm{z}$ axes of the coordinate system used by CHASM. The origin is where the shower axis intersects with the Earth's surface. Standard physics spherical coordinate conventions apply for the shower polar angle.

Figure 4 is a plot of the Cherenkov signal at the counter plane. Each pixel represents one photon counter location. The ring where the maximum signal is found forms due to the shower's Cherenkov cone at development stages near $X_{\max }$.

\section{Atmospheric Curvature and Photon Timing}

As photons propagate in the atmosphere to the counting location, they are delayed compared to the speed of light in a vacuum. For downward showers, it is sufficient to divide the delay a vertically traveling photon would experience by the cosine of its polar angle. In the context of orbital observations (nuSpaceSim), photons propagate for thousands of kilometers in the upper atmosphere before they enter the vacuum of space. As the photons get higher, their angle of propagation with respect to the atmosphere becomes steeper compared to their polar angle with 

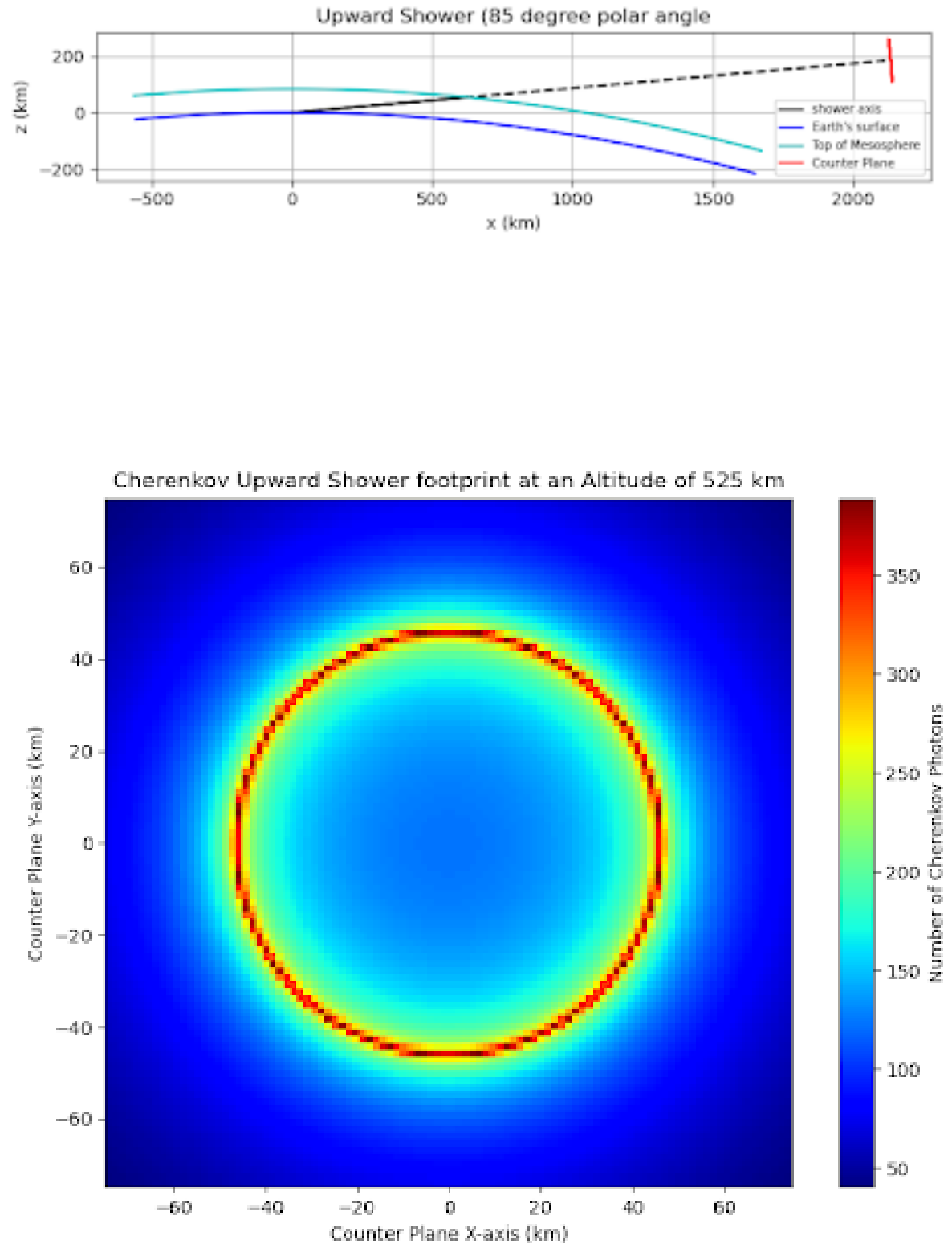

Figure 4: Top: Diagram of an upward air shower axis showing the orientation of an orbital counter array. Bottom: Cherenkov light signal at an orbital counter array normal to the shower axis at an altitude of 525 $\mathrm{km}$. 

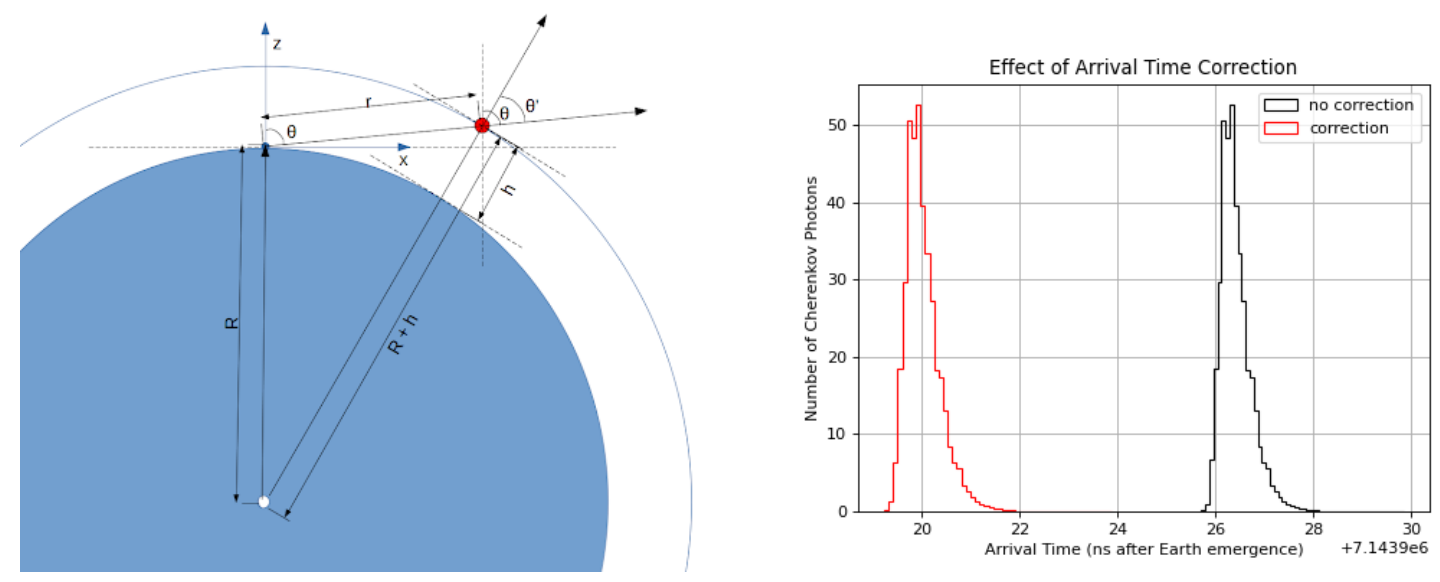

Figure 5: Left: Diagram of the geometry used to account for the curvature of the atmosphere in upward shower timing calculations. Right: Comparison of arrival time distribution when atmospheric curvature is neglected and accounted for.

respect to the original z-axis. This difference was calculated as a function of atmospheric height via the law of cosines on the inscribed triangle in Figure 5 and equation 1.

For the upward shower shown in Figures 4 and ??, there is about a seven nanosecond delay if the curvature of the atmosphere is neglected. Figure 5 shows the both the original and corrected arrival time distribution at one of the counters in the ring peak.

$$
\cos \theta^{\prime}=\frac{h^{2}+r^{2}+2 R h}{2 r(R+h)}
$$

\section{Discussion}

While comparisons between our model and CORSIKA are consistent for most regimes, we see disagreements in total Cherenkov production close (within $50 \mathrm{~m}$ for downward showers) to the center of the shower's footprint, as well as inconsistent arrival times for photons produced in early shower stages. Perturbations to the model are necessary to compensate for these differences, which mainly result from our approximation of Cherenkov production being localized to the shower axis. In reality, secondary charged particles spread out in space, and those travelling in between the axis and a counter will generate a light signal slightly sooner than our model predicts. Future iterations of CHASM will contain methods for smoothing out these inconsistencies.

\section{References}

[1] S. Lafebre, et al., Astropart. Phys. 31 (2009) 243.

[2] M. Giller, et al., J. Phys. G 30 (2004) 97.

[3] J. Krizmanic, et al., PoS(ICRC2019)936. 


\section{Full Authors List: Telescope Array Collaboration}

R.U. Abbasi ${ }^{1}$, M. Abe ${ }^{2}$, T. Abu-Zayyad ${ }^{1,3}$, M. Allen $^{3}$, Y. Arai ${ }^{4}$, E. Barcikowski ${ }^{3}$, J.W. Belz ${ }^{3}$, D.R. Bergman ${ }^{3}$, S.A. Blake ${ }^{3}$, I. Buckland ${ }^{3}$, R. Cady $^{3}$, B.G. Cheon ${ }^{5}$, J. Chiba ${ }^{6}$, M. Chikawa ${ }^{7}$, T. Fujii ${ }^{8}$, K. Fujisue ${ }^{7}$, K. Fujita ${ }^{4}$, R. Fujiwara ${ }^{4}$, M. Fukushima ${ }^{7,9}$, R. Fukushima ${ }^{4}$, G. Furlich ${ }^{3}$, R. Gonzalez ${ }^{3}$, W. Hanlon ${ }^{3}$, M. Hayashi ${ }^{10}$, N. Hayashida ${ }^{11}$, K. Hibino ${ }^{11}$, R. Higuchi ${ }^{7}$, K. Honda ${ }^{12}$, D. Ikeda ${ }^{11}$, T. Inadomi ${ }^{13}$, N. Inoue ${ }^{2}$, T. Ishii ${ }^{12}$, H. Ito ${ }^{14}$, D. Ivanov ${ }^{3}$, H. Iwakura ${ }^{13}$, H.M. Jeong ${ }^{15}$, S. Jeong ${ }^{15}$, C.C.H. Jui ${ }^{3}$, K. Kadota ${ }^{16}$, F. Kakimoto ${ }^{11}$, O. Kalashev ${ }^{17}$, K. Kasahara ${ }^{18}$, S. Kasami ${ }^{19}$, H. Kawai ${ }^{20}$, S. Kawakami ${ }^{4}$, S. Kawana ${ }^{2}$, K. Kawata ${ }^{7}$, E. Kido ${ }^{14}$, H.B. Kim ${ }^{5}$, J.H. Kim ${ }^{3}$, J.H. Kim ${ }^{3}$, M.H. Kim ${ }^{15}$, S.W. Kim ${ }^{15}$, Y. Kimura ${ }^{4}$, S. Kishigami ${ }^{4}$, Y. Kubota ${ }^{13}$, S. Kurisu ${ }^{13}$, V. Kuzmin ${ }^{17}$, M. Kuznetsov ${ }^{17,21}$, Y.J. Kwon ${ }^{22}$, K.H. Lee ${ }^{15}$, B. Lubsandorzhiev ${ }^{17}$, J.P. Lundquist ${ }^{3,23}$, K. Machida ${ }^{12}$, H. Matsumiya ${ }^{4}$, T. Matsuyama ${ }^{4}$, J.N. Matthews ${ }^{3}$, R. Mayta ${ }^{4}$, M. Minamino ${ }^{4}$, K. Mukai ${ }^{12}$, I. Myers ${ }^{3}$, S. Nagataki ${ }^{14}$, K. Nakai ${ }^{4}$, R. Nakamura ${ }^{13}$, T. Nakamura ${ }^{24}$, T. Nakamura ${ }^{13}$, Y. Nakamura ${ }^{13}$, A. Nakazawa ${ }^{13}$, T. Nonaka ${ }^{7}$, H. Oda ${ }^{4}$, S. Ogio ${ }^{4,25}$, M. Ohnishi ${ }^{7}$, H. Ohoka ${ }^{7}$, Y. Oku ${ }^{19}$, T. Okuda ${ }^{26}$, Y. Omura ${ }^{4}$, M. Ono ${ }^{14}$, R. Onogi ${ }^{4}$, A. Oshima ${ }^{4}$, S. Ozawa ${ }^{27}$, I.H. Park ${ }^{15}$, M. Potts ${ }^{3}$, M.S. Pshirkov ${ }^{17,28}$, J. Remington ${ }^{3}$, D.C. Rodriguez ${ }^{3}$, G.I. Rubtsov ${ }^{17}$, D. Ryu ${ }^{29}$, H. Sagawa ${ }^{7}$, R. Sahara ${ }^{4}$, Y. Saito ${ }^{13}$, N. Sakaki ${ }^{7}$, T. Sako ${ }^{7}$, N. Sakurai ${ }^{4}$, K. Sano ${ }^{13}$, K. Sato ${ }^{4}$, T. Seki ${ }^{13}$, K. Sekino ${ }^{7}$, P.D. Shah ${ }^{3}$, Y. Shibasaki1 ${ }^{13}$, F. Shibata ${ }^{12}$, N. Shibata ${ }^{19}$, T. Shibata ${ }^{7}$, H. Shimodaira ${ }^{7}$, B.K. Shin ${ }^{29}$, H.S. Shin ${ }^{7}$, D. Shinto ${ }^{19}$, J.D. Smith ${ }^{3}$, P. Sokolsky ${ }^{3}$, N. Sone ${ }^{13}$, B.T. Stokes ${ }^{3}$, T.A. Stroman ${ }^{3}$, T. Suzawa ${ }^{2}$, Y. Takagi $^{4}$, Y. Takahashi $^{4}$, M. Takamura ${ }^{6}$, M. Takeda $^{7}$,

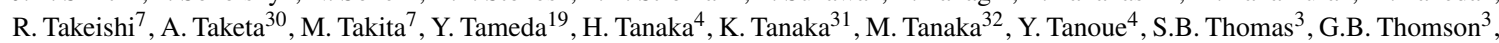
P. Tinyakov ${ }^{17,21}$, I. Tkachev ${ }^{17}$, H. Tokuno ${ }^{33}$, T. Tomida ${ }^{13}$, S. Troitsky ${ }^{17}$, R. Tsuda ${ }^{4}$, Y. Tsunesada ${ }^{4,25}$, Y. Uchihori ${ }^{34}$, S. Udo $^{11}$, T. Uehama ${ }^{13}$, F. Urban ${ }^{35}$, T. Wong ${ }^{3}$, K. Yada ${ }^{7}$, M. Yamamoto ${ }^{13}$, K. Yamazaki ${ }^{11}$, J. Yang ${ }^{36}$, K. Yashiro ${ }^{6}$, F. Yoshida ${ }^{19}$, Y. Yoshioka ${ }^{13}$, Y. Zhezher ${ }^{7,17}$, and Z. Zundel ${ }^{1}{ }^{1}$ Department of Physics, Loyola University Chicago, Chicago, Illinois, USA

2 The Graduate School of Science and Engineering, Saitama University, Saitama, Saitama, Japan

${ }^{3}$ High Energy Astrophysics Institute and Department of Physics and Astronomy, University of Utah, Salt Lake City, Utah, USA

${ }^{4}$ Graduate School of Science, Osaka City University, Osaka, Osaka, Japan

${ }^{5}$ Department of Physics and The Research Institute of Natural Science, Hanyang University, Seongdong-gu, Seoul, Korea

${ }^{6}$ Department of Physics, Tokyo University of Science, Noda, Chiba, Japan

${ }^{7}$ Institute for Cosmic Ray Research, University of Tokyo, Kashiwa, Chiba, Japan

${ }^{8}$ The Hakubi Center for Advanced Research and Graduate School of Science, Kyoto University, Kitashirakawa-Oiwakecho, Sakyo-ku, Kyoto, Japan

${ }^{9}$ Kavli Institute for the Physics and Mathematics of the Universe (WPI), Todai Institutes for Advanced Study, University of Tokyo, Kashiwa, Chiba, Japan

${ }^{10}$ Information Engineering Graduate School of Science and Technology, Shinshu University, Nagano, Nagano, Japan

${ }^{11}$ Faculty of Engineering, Kanagawa University, Yokohama, Kanagawa, Japan

${ }^{12}$ Interdisciplinary Graduate School of Medicine and Engineering, University of Yamanashi, Kofu, Yamanashi, Japan

${ }^{13}$ Academic Assembly School of Science and Technology Institute of Engineering, Shinshu University, Nagano, Nagano, Japan

${ }^{14}$ Astrophysical Big Bang Laboratory, RIKEN, Wako, Saitama, Japan

${ }^{15}$ Department of Physics, Sungkyunkwan University, Jang-an-gu, Suwon, Korea

${ }^{16}$ Department of Physics, Tokyo City University, Setagaya-ku, Tokyo, Japan

${ }^{17}$ Institute for Nuclear Research of the Russian Academy of Sciences, Moscow, Russia

${ }^{18}$ Faculty of Systems Engineering and Science, Shibaura Institute of Technology, Minato-ku, Tokyo, Japan

${ }^{19}$ Department of Engineering Science, Faculty of Engineering, Osaka Electro-Communication University, Neyagawa-shi, Osaka, Japan

${ }^{20}$ Department of Physics, Chiba University, Chiba, Chiba, Japan

${ }^{21}$ Service de Physique ThÃ $\odot$ orique, Universit $\tilde{A} \odot$ Libre de Bruxelles, Brussels, Belgium

${ }^{22}$ Department of Physics, Yonsei University, Seodaemun-gu, Seoul, Korea

${ }^{23}$ Center for Astrophysics and Cosmology, University of Nova Gorica, Nova Gorica, Slovenia

${ }^{24}$ Faculty of Science, Kochi University, Kochi, Kochi, Japan

${ }^{25}$ Nambu Yoichiro Institute of Theoretical and Experimental Physics, Osaka City University, Osaka, Osaka, Japan

${ }^{26}$ Department of Physical Sciences, Ritsumeikan University, Kusatsu, Shiga, Japan

${ }^{27}$ Quantum ICT Advanced Development Center, National Institute for Information and Communications Technology, Koganei, Tokyo, Japan

${ }^{28}$ Sternberg Astronomical Institute, Moscow M.V. Lomonosov State University, Moscow, Russia

${ }^{29}$ Department of Physics, School of Natural Sciences, Ulsan National Institute of Science and Technology, UNIST-gil, Ulsan, Korea

${ }^{30}$ Earthquake Research Institute, University of Tokyo, Bunkyo-ku, Tokyo, Japan

${ }^{31}$ Graduate School of Information Sciences, Hiroshima City University, Hiroshima, Hiroshima, Japan

32 Institute of Particle and Nuclear Studies, KEK, Tsukuba, Ibaraki, Japan

${ }^{33}$ Graduate School of Science and Engineering, Tokyo Institute of Technology, Meguro, Tokyo, Japan

${ }^{34}$ Department of Research Planning and Promotion, Quantum Medical Science Directorate, National Institutes for Quantum and Radiological Science and Technology, Chiba, Chiba, Japan

${ }^{35}$ CEICO, Institute of Physics, Czech Academy of Sciences, Prague, Czech Republic

${ }^{36}$ Department of Physics and Institute for the Early Universe, Ewha Womans University, Seodaaemun-gu, Seoul, Korea 